\title{
SHOULD A SUPPLIER ENGAGE IN THE MARKETPLACE? AN EQUILIBRIUM ANALYSIS IN A HYBRID-FORMAT SUPPLY CHAIN
}

\author{
YOU ZHAO@ AND RUI HOU*®
}

\begin{abstract}
Recently, e-commerce platforms have been acting as both a reseller and a marketplace to serve consumers. This study considers a hybrid-format supply chain that consists of a supplier who sells a product through an intermediary by a wholesale price contract. In addition, the supplier can decide whether to accept the intermediary's offer to engage in the marketplace (if the supplier accepts, then the agency fee should be paid to the intermediary). We develop a game-theoretic model to investigate the impact of supplier encroachment on the supply chain members. Then, we extend our basic model to check the robustness of our main results. Our results show that the supplier prefers to engage in the marketplace if the agency fee is low and the order fulfillment cost is relatively low, the intermediary benefits from the supplier encroachment if the agency fee is relatively low, and consumers always benefit from encroachment. Interestingly, we show that an increase in channel substitutability leads to raising the supplier's willingness to adopt an encroachment strategy. Our findings contribute to the online marketplace literature by providing valuable insights into the operation management of online marketplaces.
\end{abstract}

Mathematics Subject Classification. 90B50, 91A80.

Received February 19, 2021. Accepted August 18, 2021.

\section{Highlights}

- We focus on the supplier's encroachment decision and study its impact on supply chain members in a hybrid-format online marketplace.

- The supplier may prefer to engage in an online marketplace when the agency fee and the order fulfillment cost are within a certain range.

- The supplier's willingness to adopt an encroachment strategy is contingent on the channel substitutability and the order fulfillment cost.

\section{INTRODUCTION}

With the development of information technology and the consequent growth in the popularity of the Internet, every aspect of life has been undergoing radical changes. For example, shopping via online trading platforms, that is online intermediaries, such as "Taobao," "Amazon," and "Tmall," has greatly facilitated our everyday

Keywords. Online marketplace, supplier encroachment, channel substitutability, hybrid-format supply chain.

School of Management, Guangdong University of Technology, Guangzhou, Guangdong 510520, P.R. China.

*Corresponding author: hour@gdut.edu.cn 
activities $^{1}$. Traditionally, an online intermediary platform purchases a product at a wholesale price and resells it to the end market (e.g., BsetBuy.com) at a retail price. However, recently, intermediary platforms have been providing an online marketplace to the supplier to sell their products directly; if the supplier accepts this offer, then the agency fee is paid to the intermediary (e.g., Zappos.com). However, in reality, many suppliers not only sell their products indirectly through reselling channels, but also participate in the marketplace established by the intermediaries. For example, consider that six major publishers sell e-books through an agency mode, while Amazon simultaneously distributes e-books through a wholesale mode with the help of the Internet ${ }^{2}$. One of the biggest boosters behind Amazon's e-book giant is its control over the most popular e-book delivery method, the Kindle. As a result, Amazon not only controls e-books, but also the way people read e-books, thus further increasing its market share ${ }^{3}$.

Importantly, order fulfillment costs such as transportation cost, pick and pack fees, and inventory cost should be taken into consideration in such situations ${ }^{4}$. Wall Street Journal reports that fulfillment cost operations can run as high as $25 \%$ of the sales revenue. It is worth observing that in this case, the intermediary is required to bear the order fulfillment costs in the reselling channel, whereas the supplier has to bear the order fulfillment cost in the agency selling channel when the intermediary's offer is accepted.

It is a popular way for suppliers to increase their profits by establishing a direct selling channel. However, when the supplier decides to establish a direct selling channel in the supply chain, it will eventually compete with the retailer's selling channel; this may damage the relationship between the supplier and the retailer. For example, clothing firms such as Ralph Lauren, the Armani Group, Hugo Boss, and Baravade have established their offline or department stores. However, the existing literature has not explored the impact of the supplier's engagement in the marketplace, which is a new way to gain more profits, on the supply chain members and consumers in a hybrid-format supply chain. To fill this literature gap, we address the following research questions.

- Should a supplier engage in the marketplace?

- Can an intermediary benefit from the supplier's encroachment?

To answer these questions, we develop a game-theorical model to study the wholesale price and quantity decisions in a hybrid-format supply chain that consists of a supplier who sell products through an intermediary, and an intermediary who acts as a reseller as well as a marketplace. The timeline of the game is as follows. First, the supplier decides whether to engage in the marketplace or not and accordingly determines the wholesale price. Second, the intermediary chooses its retail quantity. Third, the supplier determines its retail quantity if it is engaged in the marketplace. Two scenarios are considered in this case: (i) the benchmark case of nonencroachment scenario in which the supplier rejects the intermediary's offer, and (ii) an encroachment scenario in which the supplier engages in the marketplace.

Our major findings can be summarized as follows. First, we find that the supplier prefers to engage in the marketplace if the agency fee is low and the order fulfillment cost is relatively low. Moreover, the intermediary benefits from the supplier encroachment if the agency fee is relatively low. Furthermore, it is observed that

\footnotetext{
${ }^{1}$ From 2014 to 2018 , the growth rate of the transaction scale in China's online shopping market slowed down by $70 \%$ as compared to its value in 2012. In 2018, the transaction scale of China's online shopping market was 7.90 trillion yuan, which was a year-on-year increase of $27.27 \%$. Moreover, in the first half of 2019 , the transaction volume of China's online retail market reached 4.34 trillion yuan, which was a year-on-year increase of 24.80\%. Source: https://www. sohu.com/a/297538822_473133 (accessed by Jun. 1. 2021).

${ }^{2}$ Amazon controls $70 \%$ of e-book sales and wants to sell e-books wholesale. In theory, they would pay the publisher a predetermined price, usually half the retail price of the e-book, and then sell it at the price they like. In addition, Amazon can sell e-books for less than it pays publishers. The retail giant is unique in book sales because of its ability to offer big discounts, even at a loss. Therefore, Amazon has realized customer loyalty and improved customer awareness; In return, these customers can buy e-books and other products from Amazon.

${ }^{3}$ What is the agency model? It's a Book Pricing System in which publishers set book prices and distributors (like Amazon or apple) charge specific fees (about 30\%) from publishers. Therefore, our problem is how to choose the most suitable model between the agency model and the wholesale model. Wholesale pricing has traditionally been used to print books. In this mode, publishers sell books to publishers at a predetermined price, usually $50 \%$ of the retail price; As a result, publishers sell the books to the public at a price they like. Source: http://publishingtrendsetter.com/industryinsight/simple-explanation-agency-model/ (accessed by Jan. 19. 2021).

${ }^{4}$ Order fulfillment by Amazon: https://www.amazon.ca/fulfillment-fees/b?node=13718757011 (accessed by Jan. 19. 2021)
} 
the consumers always benefit from encroachment. Hence, a "win-win" outcome can be achieved between the supplier and the intermediary. Second, our results suggest that the agency fee threshold decreases in order fulfillment cost, while it increases in channel substitutability. Therefore, a higher order fulfillment cost reduces the willingness of supplier encroachment, whereas a higher channel substitutability raises it. Third, we show that the equilibrium profits for the supplier, intermediary, and entire supply chain are all monotonically decreasing in channel substitutability but increasing in agency fee. In addition, we extend our basic model of simultaneous encroachment and price competition to verify the robustness of our findings. Specifically, in the case of simultaneous encroachment, we find that the supplier is more likely to encroach under sequential decisions rather than simultaneous quantity decisions; under price competition, we show that the intermediary benefits from supplier encroachment if the agency fee is moderate and the channel substitutability is low, or both the agency fee and the channel substitutability are relatively high.

The remainder of this paper is organized as follows. Section 2 presents a brief review on the existing literature. We develop a standard model in Section 3. In Section 4, we present our results, identify the conditions under which the supplier should engage in the marketplace in a hybrid-format supply chain, and evaluate the consumer surplus and social welfare. We also present a few numerical examples in this regard. Section 5 extends our basic model to examine the robustness of our major findings and discusses their implications. Finally, Section 6 summarizes the conclusions of the study, offers managerial insights, and discuss the scope for future research.

\section{Literature REVIEW}

This section analyzes the existing literature on channel structure choice and supplier encroachment.

Our study is based on channel structure choice. In this context, Ryan et al. [19] consider a company that sells its products directly in an online market and a retailer who only sells products through its own website; however, the retailer can choose to sign a contract with Amazon and sell products through its marketing system. Therefore, the retailer's available market is expanded by marketing products, but certain costs such as fixed participation fees or revenue sharing requirements have to be incurred in the process. Mantin et al. [17] provide insights into the growing popularity of the 3P market. They show that retailers create an "external choice" and improve their bargaining position in their negotiations with manufacturers by promising to have an active 3P market; this contributes to the growing popularity of this market. Hagiu and Wright [9] find that whether the market model is more popular than the reseller model or not depends on who between the independent supplier and the middleman holds more important information about the best customization of the marketing activities for each specific product. Abhishek et al. [1] employed a stylized theoretical model to answer the following question in this context: when should e-retailers adopt the agent sales model instead of the traditional resale model? They find that agent selling is more effective than resale and leads to lower retail prices; however, the e-retailer finally transfers its control of the retail price to the manufacturer. Accordingly, Tian et al. [22] find that the competition among upstream suppliers severely mitigates this possibility; moreover, the interaction between order fulfillment costs and upstream competition intensity can regulate the optimal mode selection of intermediaries. Tan and Carrillo [21] formulated and analyzed a vertical differentiated commodity model to draw a comparison between the agency model and the wholesale model. They confirm that (a) the revenue sharing structure and (b) price control by upstream publishers contribute to the benefits of the agency model. Belhadj et al. [4] examined the strategic interaction between sellers and more informative platforms in a signaling game. They find that the platform can choose between two different business models, that is, a distributor or a pure market between buyers and sellers. The market model considers a spillover effect between the internal platform sales and the seller's direct sales. This model is always preferred in the case of low-value goods. Based on the aforementioned studies, Alaei et al. [2] consider a model with two manufacturers and one e-retailer; here, the manufacturers have their web store channel and are willing to use another channel, that is, a reseller or a marketplace. Additionally, the manufacturers offer return policies in their online store channels as a competitive strategy to attract more customers. They find that this has no influence on the choice of the manufacturer between the market and reseller channels; however, it affects the profit gained by the manufacturer 
in each channel. Jia and Li [12] consider a closed-loop supply chain consisting of online markets, that is platform services, and self-owned stores for e-retailers, which are available options for upstream manufacturers to sell new and remanufactured products. Unlike these studies, we consider a hybrid-format supply chain with a supplier and an intermediary. Additionally, the intermediary acts not only as a reseller, but also as an agency seller if the supplier accepts the intermediary's offer.

This study is also related to the case of supplier encroachment in a supply chain (see e.g., [5,13-16,23]). Arya et al. [3] show that encroachment is beneficial to retailers when no synergies are admitted by suppliers. This is because the firms adopting an encroachment strategy prefer to decrease the wholesale prices when the competition between two channels increases, thereby mitigating the double marginalization problems. Accordingly, Li et al. [13] studied the supplier encroachment problem in which the retailer may be better than the supplier who is informed. They find that the retail quantity is reduced when the market size is small because of the high-cost signaling of the retailer incurred by the established supplier's channel, which aggravates the double marginalization problem. Ha et al. [8] investigated a one-to-one supply chain with supplier encroachment where the product quality is considered to be endogenous. They show that the retailer always benefits from supplier encroachment. In addition, the supplier prefers to sell the high-quality product through its own channel when quality-differentiated products are offered. Further, Zhang et al. [25] study the supplier encroachment problem with endogenous product quality decision under asymmetric information. They find that when the entry cost is intermediate, encroachment results in a lower product quality. Moreover, the retailer prefers to share information with its supplier to reduce order risk when the supplier is efficient. Guan et al. [7] develop a dynamic model to study the interaction between a strategic inventory and supplier encroachment in the second period. They show that the retailer prefers to withhold the inventory in certain cases, making the supplier less resistant to encroachment. Furthermore, in certain scenarios, the retailer may obtain more profits in the simultaneous case compared to the sequential case. However, this study considers that the supplier encroaches the end market by accepting the intermediary's offer and pays an agency fee to the intermediary; this has not been analyzed in prior studies.

\section{Model DeVElopment}

We consider a hybrid-format supply chain in which a supplier (referred to as "S") sells a product at the wholesale price $w$ with a quality level $m$ to an intermediary (referred to as "I"). In line with Tian et al. [22], we assume that the intermediary acts not only as a reseller, but also as a marketplace. Specifically, the intermediary offers an agency fee $\lambda, 0 \leq \lambda \leq 1$ to the supplier, such that the supplier must pay $\lambda$ to the intermediary on accepting this arrangement ${ }^{5}$. Therefore, we can consider the agency fee as the cost of supplier encroachment. Moreover, similar to Fleischmann et al. [6] and Tian et al. [22], we assume that the order fulfillment cost, such as delivering physical products to consumers, which is commonly cited as one of the most expensive and critical operations of online sellers, to be a fixed item to fulfill the consumers' order. Further, the intermediary will incur a fixed cost $F_{I}>0$ upon acting as a reseller; whereas, if the supplier will incur a fixed cost $F_{S}>0$ upon accepting the intermediary's offer. However, we assume that the order fulfillment cost structure is $F_{S}=F_{I}=F$. It is worth noting that the intermediary's selling cost and the supplier's unit cost for producing the product are normalized to zero. Figure 1 shows the channel structures.

According to Singh and Vives [20] and Huang et al. [11], a representative consumer's utility function is given by

$$
U\left(q_{I}, q_{S}\right)=a\left(q_{I}+q_{S}\right)-\frac{1}{2}\left(q_{I}^{2}+2 b q_{I} q_{S}+q_{S}^{2}\right)-\left(p_{I} q_{I}+p_{S} q_{S}\right)
$$

\footnotetext{
${ }^{5}$ This arrangement is commonly observed in e-commerce platforms such as JD.com and Amazon. Moreover, the supplier has to pay a fixed fee to the intermediary on accepting this arrangement; however, in line with Abhishek et al. [1], Tan and Carrillo [21], Tian et al. [22], Belhadj et al. [4] and Alaei et al. [2], it is normalized to zero. Empirical data show that the values of the agency fees range from $6 \%$ to $25 \%$ and $5 \%$ to $12 \%$ of the retail price on Amazon.com and JD.com, respectively.
} 
Non-encroachment

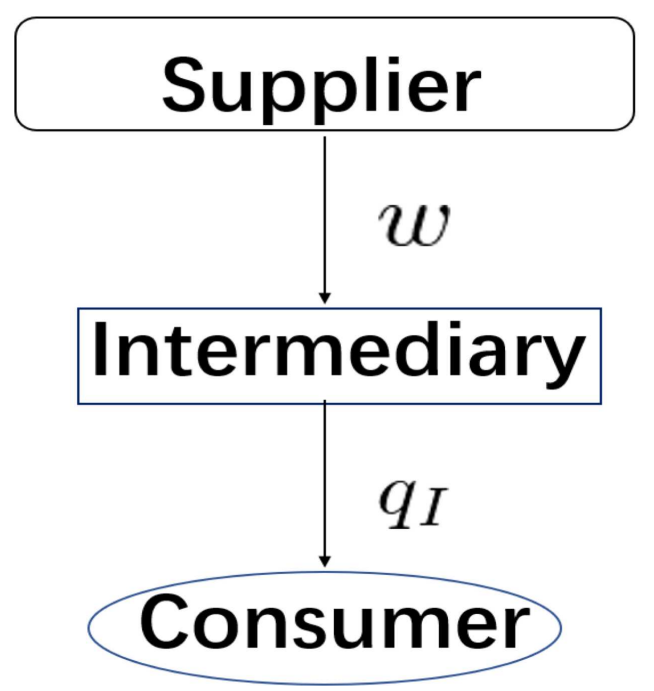

Encroachment



FiguRE 1. Non-encroachment and encroachment.

where $a$ represents the basic market demand, which is a non-negative item. $q_{I}\left(p_{I}\right)$ and $q_{S}\left(p_{S}\right)$ are the intermediary's and supplier's retail quantities (prices), respectively. $b \in(0,1)$ represent channel substitutability with a higher value of $b$ leading to a greater intensity of quantity competition. By solving the first-order conditions $\frac{\partial U\left(q_{I}, q_{S}\right)}{\partial q_{I}}=0$ and $\frac{\partial U\left(q_{I}, q_{S}\right)}{\partial q_{S}}=0$, the inverse demand functions in the reselling and agency channels, respectively, can be expressed as follows.

$$
\begin{aligned}
& p_{I}=a-q_{I}-b q_{S}, \\
& p_{S}=a-q_{S}-b q_{I} .
\end{aligned}
$$

The above inverse demand functions are widely adopted in operations management (see e.g., [3,11]). Moreover, similar to the non-encroachment scenario, the inverse demand function in the reselling channel is given by $p_{I}=a-q_{I}$.

Based on the above description, the timeline of the game is as follows (see Fig. 2). First, the supplier decides whether to engage in the marketplace and then determines the wholesale price $w$. Second, the intermediary chooses the retail quantity $q_{I}$. Third, the supplier determines the retail quantity $q_{S}$ upon engaging in the marketplace. We employ the backward induction method to derive the equilibrium outcomes of this game. The timeline of the game is in line with Arya et al. [3]; however, given the various possibilities of the supplier's and intermediary's decision sequences, we consider a situation where the supplier and the intermediary determine the retail quantities $q_{S}$ and $q_{I}$ simultaneously to examine the robustness of the model. Additionally, we investigate the scenarios of price competition in Section 5 .

\section{Results}

In this section, first, we derive the equilibrium outcomes under the non-encroachment and encroachment scenarios, respectively. Second, we compare these two scenarios to determine when the supplier should engage in the marketplace. Third, we study the impact of supplier encroachment on consumer surplus and social welfare. 




Figure 2. Timeline of the game.

\subsection{Benchmark: non-encroachment scenario}

We consider a benchmark case where the supplier rejects the intermediary's offer, that is, a non-encroachment scenario. We solve the game by using backward induction. Here, the intermediary first maximizes the following problem:

$$
\max _{q_{I}}\left(a-q_{I}-w\right) q_{I}-F
$$

by choosing

$$
q_{I}^{N}(w)=\frac{1}{2}(a-w) .
$$

Anticipating the intermediary's reaction, the supplier maximizes the following problem

$$
\max _{w} \frac{1}{2}(a-w) w
$$

by choosing

$$
w^{N}=\frac{a}{2}
$$

We summarize equilibrium results under the non-encroachment scenario as follows.

$$
\begin{aligned}
& w^{N}=\frac{a}{2}, q_{I}^{N}=\frac{a}{4}, \\
& \prod_{I}^{N}=\frac{a^{2}}{16}-F, \prod_{S}^{N}=\frac{a^{2}}{8} .
\end{aligned}
$$

\subsection{Encroachment scenario}

We now consider a situation where the supplier accepts the intermediary's offer, that is, the supplier decides to encroach the end market. Again, the equilibrium results are derived via backward induction. For any value of the wholesale price $w$ and intermediary's retail quantity $q_{I}$, the supplier first solves the following maximization problem:

$$
\max _{q_{S}} w q_{I}+(1-\lambda)\left(a-b q_{I}-q_{S}\right) q_{S}-F
$$

by choosing

$$
q_{S}^{E}\left(q_{I}\right)=\frac{1}{2}\left(a-b q_{I}\right) .
$$

Anticipating the supplier's reaction, the intermediary maximizes the following problem:

$$
\max _{q_{I}}\left(a-q_{I}-b q_{S}-w\right) q_{I}+\lambda\left(a-b q_{I}-q_{S}\right) q_{S}-F,
$$


by choosing

$$
q_{I}^{E}(w)=\frac{a(b+b \lambda-2)+2 w}{b^{2}(2+\lambda)-4} .
$$

By substituting $q_{I}^{E}(w)$ into $q_{S}^{E}\left(q_{I}\right)$, the supplier chooses $w^{E}$ to maximize profits. We also summarize the equilibrium outcomes as follows.

$$
\begin{aligned}
w^{E} & =-\frac{a[8+b(-8 \lambda+b(-6+b+b \lambda(4+\lambda)))]}{2\left[-8+b^{2}(5+\lambda)\right]}, \\
q_{I}^{E} & =\frac{2 a(-1+b)}{-8+b^{2}(5+\lambda)}, \\
q_{S}^{E} & =-\frac{a[-8+b(2+b(3+\lambda))]}{2\left[-8+b^{2}(5+\lambda)\right]}, \\
\prod_{I}^{E} & =\frac{a^{2}\left[16-32 b+64 \lambda+8 b^{3}(2+\lambda)-4 b^{2}\left(-2+21 \lambda+4 \lambda^{2}\right)+b^{4}\left(-8+21 \lambda+10 \lambda^{2}+\lambda^{3}\right)\right]}{4\left[-8+b^{2}(5+\lambda)\right]^{2}}-F, \\
\prod_{S}^{E} & =\frac{a^{2}\left[-12+8 b+8 \lambda-b^{2}\left(-1+4 \lambda+\lambda^{2}\right)\right]}{4\left[-8+b^{2}(5+\lambda)\right]}-F .
\end{aligned}
$$

Based on the above equilibrium results, we obtain the observations stated in the following lemma.

Lemma 4.1. The following relationships always hold: $w^{E}<w^{N}, q_{I}^{E}<q_{S}^{E}$.

By Lemma 4.1, we find that $w^{E}<w^{N}$ always holds, that is, the wholesale price under the encroachment scenario is lower than that under the non-encroachment scenario. Hence, if the supplier engages in the marketplace, the wholesale price will be reduced, and the double marginalization problem will be mitigated. Moreover, we find that $q_{I}^{E}<q_{S}^{N}$ always holds, that is, the retail quantity under reselling channel is always lower than that under the non-encroachment scenario. This is because when the supplier engages in the marketplace, the selling price in the agency selling channel will be always lower than that in the reselling channel.

\subsection{Encroachment vs. non-encroachment}

Using the above equilibrium outcomes, we answer the question of whether the supplier should encroach or not, or the supply chain members benefit from the supplier' encroachment, as stated in the following theorem.

Theorem 4.2. In equilibrium, for any $b \in(0,1)$

(i) when $F \leq F_{0}=\frac{a^{2}\left[-12+8 b+8 \lambda-b^{2}\left(-1+4 \lambda+\lambda^{2}\right)\right]}{4\left[-8+b^{2}(5+\lambda)\right]^{2}}-\frac{a^{2}}{8}$, the supplier prefers to encroach if and only if $\lambda \in\left(0, \lambda_{0}(b, F)\right)$;

(ii) the intermediary is beneficial to the supplier's encroachment if and only if $\lambda \in\left(0, \lambda_{1}(b)\right)$, where $\lambda_{0}(b, F)=$ $\frac{16 a^{2}-9 a^{2} b^{2}-8 b^{2} F}{4 a^{2} b^{2}}-\frac{1}{4} \sqrt{\frac{256 a^{4}-416 a^{4} b^{2}+128 a^{4} b^{3}+57 a^{4} b^{4}+256 a^{2} b^{2} F-176 a^{2} b^{4} F+64 b^{4} F^{2}}{a^{4} b^{4}}}, \lambda_{1}(b)$ is the unique solution of $\frac{a^{2}\left(16-32 b+64 \lambda+8 b^{3}(2+\lambda)-4 b^{2}\left(-2+21 \lambda+4 \lambda^{2}\right)+b^{4}\left(-8+21 \lambda+10 \lambda^{2}+\lambda^{3}\right)\right)}{4\left(-8+b^{2}(5+\lambda)\right)^{2}}=\frac{a^{2}}{16}$.

Theorem 4.2 presents the conditions under which the supplier accepts the intermediary's offer and determines to encroach the end market. For any given value of $b$, part(i) reveals that the supplier will benefit from encroachment when the order fulfillment cost does not exceed a given threshold and the agency fee is relatively low. Therefore, if the supplier has the retail quantity decision power upon accepting the intermediary's offer; however, the supplier also incurs a fixed order fulfillment cost concurrently. Moreover, a smaller agency fee will elicit the supplier to accept the intermediary's offer. One may expect that the intermediary benefits from the supplier's encroachment when the supplier accepts a higher agency fee arrangement. However, part(ii) suggests 
that the intermediary is beneficial to the supplier's encroachment if the agency fee is low, since the supplier is willing to adjust to a lower wholesale price under the encroachment scenario compared to that under the non-encroachment scenario. In addition, a reduction in demand in the agency channel is less than the reduction in the wholesale price; therefore, the intermediary benefits from the supplier encroachment when the agency fee is low.

Combining part(i) and (ii), we find that a "win-win" outcome can be achieved for the supplier and the intermediary when $0<\lambda \leq \min \left\{\lambda_{0}(b, F), \lambda_{1}(b)\right\}$ holds.

Proposition 4.3. Under the encroachment scenario, in equilibrium,

(i) the equilibrium profits for the supplier, intermediary, and entire supply chain monotonically decrease with channel substitutability;

(ii) the equilibrium profits for the intermediary and the whole supply chain monotonically increase with the agency fee, whereas they decrease with it in the case of the supplier;

(iii) the threshold $\lambda_{0}(b, F)$ decreases with the order fulfillment cost $F$, while it increases with the channel substitutability $b$.

Proposition 4.3(i) indicates that an increase in the channel substitutability will decrease the profits for the supplier, intermediary, and entire supply chain. Hence, the profits of the supply chain parties decrease due to an increasing channel competition. Interestingly, part(ii) indicates that the intermediary and the whole supply chain can gain more profits as the agency fee increases, whereas the supplier is negatively affected by it, which is in sharp contrast to Tian et al. [22]. A supplier who engages in the marketplace can adjust the retail quantity and reduce the wholesale price for the intermediary to increase the demand in the reselling channel. However, the intermediary obtains new profits from the agency selling channel; therefore, the supplier, intermediary, and entire supply chain can benefit from an increase in the agency fee. The intuition is that the supplier prefers to reject the intermediary's offer as the channel substitutability increases. However, part(iii) presents that the agency fee threshold decreases in $F$, whereas it increases in $b$, that is, the increased $F$ may reduce the supplier's willingness to adopt an encroachment strategy, whereas due to an increased $b$, the supplier may prefer to encroach. This is because although the intermediary may offer a higher agency fee to the supplier when the channel substitutability increases, the supplier will still prefer to accept it when the revenue from the reselling channel outweighs the loss from the agency channel.

Overall, we find that the intermediary should focus on choosing an appropriate value of the agency fee to ensure that the supplier accepts the offer when the order fulfillment cost is bounded. Conversely, the supplier also should consider a trade-off between the order fulfillment cost and the profits gained from using the retail quantity power.

\subsection{Consumer surplus and social welfare}

We investigate the consumer surplus and social welfare under the non-encroachment and encroachment scenarios. We substitute the inverse demand functions introduced in Section 3 into equation (3.1) to derive the consumer surplus under the non-encroachment and encroachment scenarios given by $U\left(q_{I}, q_{S}\right)=\frac{1}{2}\left(q_{I}^{2}+2 b q_{I} q_{M}+q_{S}^{2}\right)$ and $U\left(q_{I}\right)=\frac{1}{2} q_{I}^{2}$, respectively. Based on the above description, the consumer surplus under the nonencroachment and encroachment scenarios are respectively given by

$$
\left\{\begin{array}{l}
\mathrm{CS}^{N}=\frac{a^{2}}{32}, \quad \text { and } \\
\mathrm{CS}^{E}=\frac{a^{2}\left(80+b^{2}[-4(27+4 \lambda)+b(4-4 \lambda+b(3+\lambda)(11+\lambda)))\right]}{8\left(-8+b^{2}(5+\lambda)\right)^{2}}
\end{array}\right.
$$


The social welfare is given by $\mathrm{SW}=\prod_{I}+\prod_{S}+\alpha \mathrm{CS}$, where $\alpha$ measures the relative importance of consumer surplus (see e.g., [11]). We summarize the social welfare considering the following two scenarios.

$$
\left\{\begin{aligned}
\mathrm{SW}^{N}= & \frac{7 a^{2}}{32}-F \\
& a^{2}\left[224+80 \alpha+b\left(-192+4 b^{2}(28-\lambda(-8+\alpha)+\alpha)\right.\right. \\
\mathrm{SW}^{E}= & \frac{\left.\left.+b^{3}(3+\lambda)(-2+11 \alpha+\lambda(2+\alpha))-4 b(30+27 \alpha+4 \lambda(3+\alpha))\right)\right]}{8\left(-8+b^{2}(5+\lambda)\right)^{2}}-2 F .
\end{aligned}\right.
$$

According to equations (4.16) and (4.17), we compare the consumer surplus and social welfare under the encroachment and non-encroachment scenarios, respectively, as stated in the following lemma.

Lemma 4.4. The following relationships hold: $\mathrm{CS}^{E}>\mathrm{CS}^{N}$ and $\mathrm{SW}^{E}>\mathrm{SW}^{N}$.

Lemma 4.4 indicates that the consumers and social welfare derive benefits from supplier encroachment. This is because when the supplier engages in the marketplace, both the supplier and intermediary suffer from channel competition due to a reduced wholesale price; this causes the intermediary to adopt flexibility and adjust the retail quantity, that is, a lower retail price will be determined under the encroachment scenario rather than the non-encroachment scenario. These results contradict Arya et al. [3], who concluded that consumers benefit from supplier encroachment under certain conditions.

The following proposition characterizes the impact of channel substitutability and agency fee on consumer surplus and social welfare, respectively.

Proposition 4.5. (i) $\frac{\partial \mathrm{CS}^{E}}{\partial b}<0, \frac{\partial \mathrm{CS}^{E}}{\partial \lambda}>0$;

(ii) $\frac{\partial \mathrm{SW}^{E}}{\partial b}<0, \frac{\partial \mathrm{SW}^{E}}{\partial \lambda}>0$.

Based on Proposition 4.5, we find that both consumer surplus and social welfare decrease with an increase in the channel substitutability, whereas they increase with an increase in the agency fee. That is because an increased channel substitutability has a negative impact on the revenues of the supplier and the intermediary, whereas an increased agency fee has a positive impact on them. These results are explained in Proposition 4.3.

\subsection{Numerical examples}

In order to explain our theoretical results, we present some numerical examples. It is worth noting that under the non-encroachment scenario, the equilibrium outcomes do not depend on the order fulfillment cost, agency fee, and channel substitutability. Therefore, we do not provide numerical examples in this case and focus on the impact of the agency fee and channel substitutability on the profits obtained by the intermediary and the supplier, and determine the consumer surplus under the encroachment scenario.

Similar to Tian et al. [22], we set $a=10$ and $F=1$, for the agency fee and channel substitutability, respectively, and the range between 0 and 1 . Figure 3 shows the impact of the agency fee and channel substitutability on the intermediary's profit. We find that the intermediary's profit increases as the agency fee increases, whereas it decreases with an increase in channel substitutability.

Figure 3 shows the impact of the agency fee and channel substitutability on the intermediary's profit. We find that the intermediary's profit increases as the agency fee increases, whereas it decreases with the increase in channel substitutability. Not surprisingly, the intermediary suffers from the loss of profit because of the high intensity competition; however, she can benefit from a high agency fee paid by the supplier simultaneously. In practice, for highly competitive products, the intermediary should induce the supplier to increase the agency fee to achieve a "win-win" outcome.

Figure 4 illustrates the impact of the agency fee and channel substitutability on the supplier's profit. Clearly, the supplier's profit decreases as both the agency fee and channel substitutability increase. In other words, the supplier is hurt by the increase in agency fee because he has to bear it, and suffer the profit loss due to high channel substitutability. In practice, the supplier may decide not to engage in the online marketplace when he 




FIGURE 3. Impact of agency fee and channel substitutability on the intermediary's profit.



FIGURE 4. Impact of agency fee and channel substitutability on the supplier's profit.

has to pay a high agency fee. Moreover, for highly competitive products, the supplier face a trade-off problem between the agency fee and channel substitutability.

Figure 5 reveals the impact of the agency fee and channel substitutability on the consumer surplus. Concretely, consumer surplus increases as the agency fee increases, whereas it decreases with the increase in channel substitutability. That is, consumers benefit from the raised agency fee charged by the intermediary, whereas the increase in channel substitutability is detrimental to them.

The aformentioned observations are in line with our obtained theoretical results. Further, we validate our model.

\section{Discussion}

This section presents some extensions to check the robustness of our basic model. First, we change the encroachment setting to examine the robustness of our main results and investigate the impact of decision sequence 


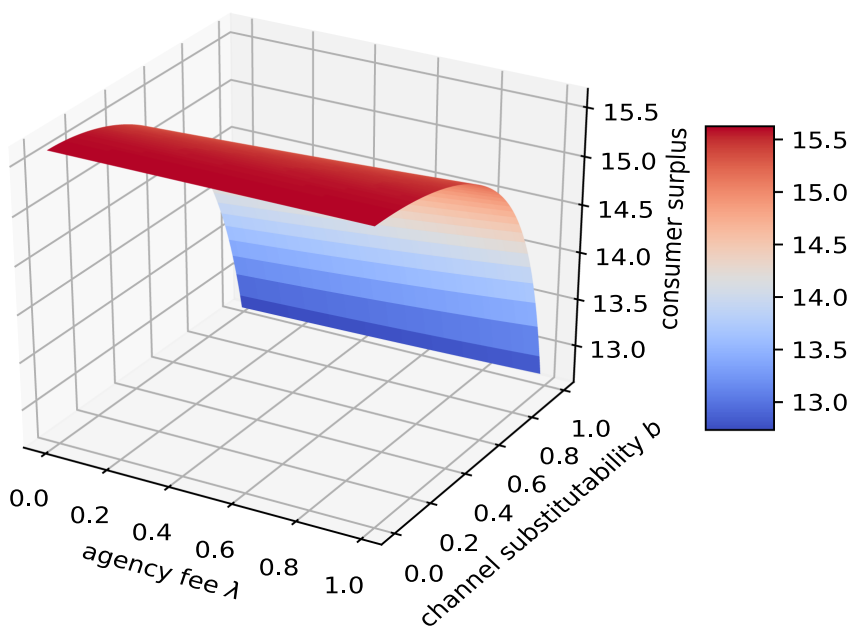

Figure 5. Impact of agency fee and channel substitutability on consumer surplus.

on the supplier's encroachment decision. Additionally, we consider that the supplier and the intermediary determine the retail quantity simultaneously. Next, since the quantity competition case has been explored in our basic model, we now consider a case where both the intermediary and the supplier encroach in price competition.

\subsection{Simultaneous encroachment}

In our basic model, we consider the decision sequence in a sequential encroachment setting wherein the intermediary determines the retailer quantity in the reselling channel before the supplier sets the retail quantity in the agency selling channel. However, other encroachment settings can be explored to test the robustness of the results. Form here, we investigate the simultaneous encroachment setting wherein the supplier and the intermediary determine the retail quantity simultaneously, which is in line with Arya et al. [3], Huang et al. [10] and Huang et al. [11].

In this setting, the analysis procedure is in line with our basic model. We summarize the results as stated in the following theorem.

Theorem 5.1. Under simultaneous encroachment, in equilibrium, for any given $b \in(0,1)$

(i) when $F \leq F_{1}=-\frac{a^{2}\left[12-8 \lambda+b\left(-8+b(1+\lambda)^{2}\right)\right]}{4\left[-8+b^{2}(3+\lambda)\right]}-\frac{a^{2}}{8}$, the supplier prefers to encroach if and only if $\lambda \in$ $\left(0, \lambda_{2}(b, F)\right)$;

(ii) the intermediary is beneficial to the supplier's encroachment if and only if $\lambda \in\left(\lambda_{3}(b), 1\right)$.

Theorem 5.1 presents conditions in which the supplier and the intermediary benefit from encroachment. These findings are somewhat similar to our main results. Part(i) suggests that the supplier engages in the marketplace if the order fulfillment cost is low and the agency fee is relatively low. It is worth noting that the threshold $\lambda_{2}(b, F)$ is different from the threshold $\lambda_{2}(b, F)$ in our main results. In contrast with our main results, part(ii) shows that the intermediary benefits from the supplier's encroachment if the agency fee is relatively high. In other words, different decision sequences affect the conditions under which the intermediary benefits from the supplier's encroachment when the supplier engages in the marketplace. Moreover, we find that if $\lambda_{2}(b)>\lambda_{3}(b)$ and $\lambda_{3}(b)<\lambda \leq \lambda_{2}(b)$, a "win-win" outcome can be achieved for both the supplier and the intermediary. Clearly, although we have changed the decision sequence to simultaneous encroachment, Theorem 5.1 indicates that the supplier does not always prefer to engage in online marketplace. That is, the supplier's encroachment decision is dependent on the order fulfillment cost and the agency fee. Moreover, the online marketplace benefits from 
the supplier's encroachment under certain conditions. These results are consistence with the results obtained in our basic model.

By comparing the sequential and simultaneous encroachment settings, the following proposition can be obtained.

Proposition 5.2. (i) The supplier is more likely to encroach under sequential than under simultaneous quantity decisions;

(ii) The intermediary is better off which the decision sequence is dependent on the relationship between $\lambda_{1}(b)$ and $\lambda_{3}(b)$.

A previous study, Ha et al. [8] has shown that the supplier prefers to establish a direct selling channel under simultaneous than sequential quantity decisions when considering the product quality level. However, based on Proposition 5.2(i), we find that the supplier is more likely to encroach under sequential than under simultaneous quantity decisions, that is, $\lambda_{1}(b, F)>\lambda_{2}(b, F)$, which is in line with Arya et al. [3]. According to part(ii), we find that it is difficult to distinguish the dominant decision sequence. That is, when we are ready to compare the values of $\lambda_{1}(b)$ and $\lambda_{3}(b)$, there are two possible outcomes: (i) $\lambda_{1}(b) \geq \lambda_{3}(b)$, that is, under different decision sequences, the intermediary benefits from the supplier's encroachment under a totally different region of the agency fee; (ii) $\lambda_{1}(b)<\lambda_{3}(b)$, in which there is a coincidence area of the agency fee between the two decision sequences. Nevertheless, we cannot determine which decision sequence is preferred by the intermediary.. This finding is in sharp contrast to Arya et al. [3].

\subsection{Price competition}

Since the quantity competition has been investigated in our basic model, here we consider the price competition between the supplier and the intermediary under the encroachment scenario. According to Arya et al. [3], Mittendorf et al. [18], Yoon [24] and Huang et al. [10], the inverse demand function can be expressed as

$$
q_{i}=\frac{a}{1+b}-\frac{1}{1-b^{2}} p_{i}+\frac{b}{1-b^{2}} p_{j} \quad \text { for } \quad i \neq j, i=I, S .
$$

The timeline of the game is in line with our basic model and the equilibrium outcomes are listed in the Appendix A. We present the findings in the following theorem.

Theorem 5.3. Under price competition, in equilibrium,

(i) when $F \leq F_{2}=-\frac{a^{2}(-1+\lambda)\left[12-8 \lambda+b\left(4-8 \lambda+b\left(-9+2 b^{2}+\lambda(4+\lambda)+b(-1+\lambda(4+\lambda))\right)\right)\right]}{4(1+b)\left(8+b^{4}-8 \lambda+b^{2}(-1+\lambda)(5+\lambda)\right)}-\frac{a^{2}}{8}$, for any given $b \in(0,1)$, the supplier prefers to encroach if and only if $\lambda \in\left(0, \lambda_{4}(b, F)\right)$;

(ii) the intermediary is beneficial to the supplier's encroachment if and only if $\lambda \in\left(\lambda_{5}(b), \lambda_{6}(b)\right)$ and $b \in\left(0, b_{2}\right)$, or $\lambda \in\left(\lambda_{7}(b), 1\right)$ and $b \in\left[b_{2}, 1\right)$.

Not surprisingly, based on Theorem 5.3(i), we observe that the supplier prefers to engage in the marketplace if both the order fulfillment cost and the agency fee are low. This result is in line with our main results as stated in our basic model. However, it is directly observed from part(ii) that the intermediary benefits from the supplier's encroachment if the agency fee is moderate and the channel substitutability is low, or if both the agency fee and the channel substitutability are relatively high. The reason is that when the channel substitutability is low, if the intermediary offers a low agency fee, it will not be the best choice; however, if she offers a high agency fee then the supplier may reject this arrangement. Conversely, when the channel substitutability is high, if the intermediary offers a low agency fee, it may hurt her revenue because the competition between the reselling channel and the agency channel will be high. Similarly, we can find that there exists a region where a "winwin" outcome can be achieved for both the supplier and the intermediary. When the online marketplace and the supplier engage in price competition, Theorem 5.3 suggests that the supplier's encroachment decision is contingent on the order fulfillment cost and the agency fee. Further, we show that a "win-win" outcome can be achieved for both the online marketplace and the supplier. In summary, our main findings obtained for the basic model still hold under the price competition scenario and the robustness of our basic model is checked. 


\section{Conclusions AND MANAgerial insights}

\subsection{Conclusions}

Motivated by the rise of intermediaries that offer online marketplaces to the supplier (e.g., Amazon, JD.com) and establish agency selling channels to sell products to consumers, we consider a hybrid supply chain consisting of a supplier and an intermediary in which the intermediary acts as both a reseller and an agency seller (if the supplier accepts her offer).

Further, this study investigates the impact of agency fee, channel substitutability, and order fulfillment cost on the supplier and the intermediary profitability. Additionally, our study explores the conditions under which the supplier should engage in the marketplace.

Our major findings are summarized as follows. First, we find that the supplier prefers to engage in the marketplace if the agency fee is low and the order fulfillment cost is relatively low, the intermediary benefits from the supplier encroachment if the agency fee is relatively low, and consumers always benefits from encroachment. In other words, a "win-win" outcome can be achieved for both the supplier and the intermediary. Second, our results suggest that the agency fee threshold decreases in order fulfillment cost, whereas increases in channel substitutability. That is, a higher order fulfillment cost reduces the supplier's willingness to adopt an encroachment strategy; however, a higher channel substitutability raises it. Third, we show that the equilibrium profits for the supplier, the intermediary, and the whole supply chain monotonically decrease with channel substitutability, whereas increase with agency fee. In addition, we extend our basic model, that is, simultaneous encroachment and price competition, to check the robustness of our findings. Concretely, under the simultaneous encroachment scenario, we find that the supplier is more likely to encroach under sequential than under simultaneous quantity decisions, while under price competition, we show that the intermediary benefits from the supplier's encroachment if the agency fee is moderate and the channel substitutability is low, or if both the agency fee and channel substitutability are relatively high.

\subsection{Managerial insights and limitations}

Our findings provide some meaningful managerial insights. First, to achieve a "win-win" outcome, online marketplaces (e.g., Amazon.com, JD.com) should charge a relatively low agency fee, and the supplier should control the cost of order fulfillment. This insight is in line with real-world situations. According to Tian et al. [22], for different products, JD.com charges an agency fee from $6 \%$ to $25 \%$ and Amazon.com charges an agency fee from $5 \%$ to $12 \%$, which fully verifies the effectiveness of our insights. Second, for highly competitive products, the supplier should accept the marketplace's offer to extract profits. However, simultaneously, a higher order fulfillment cost may reduce the supplier's willingness to adopt an encroachment strategy; thus, he may attempt to reduce the order fulfillment cost by introducing new and advanced technologies. This insight shows that the supplier's willingness encroachment to adopt an encroachment strategy is contingent on the trade-off between the channel substitutability and the order fulfillment cost. In practice, it is commonly known that JD.com has two operation modes ${ }^{6}$ : (1) JD self-operated store; (2) JD flagship store. We can observe that the supplier prefers to open a flagship store for highly competitive products, such as smartphones and computers. Third, for highly competitive products, a higher channel substitutability (agency) hurts (benefits) the supply chain members. The intuition behind this insight hinges on the trade-off between the channel substitutability and the agency fee.

In summary, the aforementioned insights show that the order fulfillment cost, channel substitutability, and agency fee are three interacting forces that direct the equilibrium decisions for supply chain members.

Nevertheless, this study has some limitations. First, we only consider a supply chain with a supplier and an intermediary; however, it will be beneficial to study two competing supply chains. Second, we assume that the

\footnotetext{
${ }^{6} \mathrm{JD}$ self-operated store: the operation is the responsibility of JD, and the business is mainly responsible for promotion, such as which advertisements to put and which activities to participate in. JD flagship store: businesses operate more by themselves, whether it is the daily operation of the store or the logistics and promotion of products, which are operated by businesses themselves.
} 
order fulfillment cost for the supplier and the intermediary are symmetric; however, in real-life situations, the asymmetric order fulfillment cost structure should be considered. Finally, since, in practice, the market demand is uncertain, information asymmetry cases shall be explored. Therefore, future studies should build on our study and extend it by investigating these issues.

\section{Appendix A.}

Proof of Lemma 4.1. Firstly, we easily have that $w^{N}-w^{E}=\frac{a b(-8 \lambda+b(-1+b+\lambda+b \lambda(4+\lambda)))}{2\left(-8+b^{2}(5+\lambda)\right)}>0$. Secondly, we can obtain that $q_{S}^{E}-q_{I}^{E}=\frac{a(-4+b(-2+b(3+\lambda)))}{2\left(-8+b^{2}(5+\lambda)\right)}>0$. This concludes the proof.

Proof of Theorem 4.2. We first show that the supplier accepts the intermediary's offer if $F \leq F_{0}$ and $\lambda<$ $\lambda_{0}(b, F)$. Concretely, we should find the sufficient conditions for $\prod_{S}^{E}>\prod_{S}^{N}$. Second, we present the intermediary benefits from the supplier encroachment if $\lambda<\lambda_{1}(b)$. Concretely, we should find the sufficient conditions for $\prod_{I}^{E}>\prod_{I}^{N}$.

Proof of Proposition 4.3. Part(i). For given any value of $a>0,1>b>0,1>\lambda>0$ and $1>\alpha>0$, we can easily have that

$$
\begin{aligned}
& \frac{\partial \prod_{I}^{E}}{\partial b}=-\frac{2 a^{2}(-1+b)\left(32+b\left(-24-12 b(1+\lambda)+b^{2}(2+\lambda)(5+\lambda)\right)\right)}{\left(-8+b^{2}(5+\lambda)\right)^{3}}<0, \\
& \frac{\partial \prod_{S}^{E}}{\partial b}=-\frac{2 a^{2}(-1+b)(-8+b(5+\lambda))}{\left(-8+b^{2}(5+\lambda)\right)^{3}}<0 .
\end{aligned}
$$

Part(ii). For given any value of $a>0,1>b>0,1>\lambda>0$ and $1>\alpha>0$, we can easily have that

$$
\begin{aligned}
& \frac{\partial \prod_{I}^{E}}{\partial \lambda}=\frac{a^{2}\left(-512-8 b^{5}(-1+\lambda)+192 b^{2}(5+\lambda)-4 b^{4}(151+\lambda(59+6 \lambda))+\right.}{4\left(-8+b^{2}(5+\lambda)\right)^{3}}>0, \\
& \frac{\partial \prod_{S}^{E}}{\partial \lambda}=-\frac{a^{2}(-8+b(2+b(3+\lambda)))(-8+b(-2+b(7+\lambda)))}{4\left(-8+b^{2}(5+\lambda)\right)^{3}}>0 .
\end{aligned}
$$

Part(iii). For given any value of $a>0,1>b>0,1>\lambda>0$ and $1>\alpha>0$, we can easily have that

$$
\begin{aligned}
& \frac{\partial \lambda_{0}(b, F)}{\partial F}=-\frac{2}{a^{2}}+\frac{a^{2}\left(-32+22 b^{2}\right)-16 b^{2} F}{a^{4} b^{2} A}<0, \\
& \frac{\partial \lambda_{0}(b, F)}{\partial b}=\frac{64 b^{2} F+8 a^{2}\left(16+2 b^{3}-b^{2}(13+A)\right)}{a^{2} b^{5} A}>0
\end{aligned}
$$

where, $A=\sqrt{57-\frac{176 F}{a^{2}}+32\left(\frac{8}{b^{4}}+\frac{4}{b}+\frac{2 F^{2}}{a^{4}}+\frac{-13+\frac{8 F}{a^{2}}}{b^{2}}\right)}$.

Proof of Lemma 4.4. For given any value of $a>0,1>b>0,1>\lambda>0$ and $1>\alpha>0$, first, we can easily have that

$$
\mathrm{CS}^{E}-\mathrm{CS}^{N}=\frac{a^{2}}{32}\left(\frac{4\left(80+b^{2}(-4(27+4 \lambda)+b(4-4 \lambda+b(3+\lambda)(11+\lambda)))\right)}{\left(-8+b^{2}(5+\lambda)\right)^{2}}-1\right)>0
$$


Second, we can also obtain that

$$
\mathrm{SW}^{E}-\mathrm{SW}^{N}=\frac{a^{2}}{32}\left(\begin{array}{l}
4\left(224+80 \alpha+b\left(-192+4 b^{2}(28-\lambda(-8+\alpha)+\alpha)+b^{3}(3+\lambda)\right.\right. \\
\frac{(-2+11 \alpha+\lambda(2+\alpha))-4 b(30+27 \alpha+4 \lambda(3+\alpha))))}{\left(-8+b^{2}(5+\lambda)\right)^{2}}-7
\end{array}\right)>0 .
$$

This concludes the proof.

Proof of Proposition 4.5. For given any value of $a>0,1>b>0,1>\lambda>0$ and $1>\alpha>0$, first, we have that

$$
\begin{aligned}
& \frac{\partial \mathrm{CS}^{E}}{\partial b}=\frac{a^{2} b(-16(-2+\lambda)+b(24(-1+\lambda)+b(6-18 \lambda+b(-1+\lambda)(5+\lambda))))}{2\left(-8+b^{2}(5+\lambda)\right)^{3}}>0, \\
& \frac{\partial \mathrm{CS}^{E}}{\partial \lambda}=-\frac{a^{2}(-1+b) b^{2}\left(-8+b^{2}(6+b(-1+\lambda))\right)}{2\left(-8+b^{2}(5+\lambda)\right)^{3}}>0 .
\end{aligned}
$$

Second, we also have that

$$
\begin{gathered}
\frac{\mathrm{SSW}^{E}}{\partial b}=\frac{\alpha)-16 b(40-2 \alpha+\lambda(2+\alpha))+2 b^{3}(\lambda(74+4 \lambda-9 \alpha)+3(58+\alpha))}{2\left(-8+b^{2}(5+\lambda)\right)^{3}}>0, \\
\frac{\partial \mathrm{SW}^{E}}{\partial \lambda}=\frac{a^{2}(-1+b) b^{2}\left(4(-1+b)\left(-4+b^{2}(2+\lambda)\right)+\left(8+b^{2}(-6+b-b \lambda)\right) \alpha\right)}{2\left(-8+b^{2}(5+\lambda)\right)^{3}}>0 .
\end{gathered}
$$

This concludes the proof.

Proof of Theorem 5.1. We now present the equilibrium outcomes under simultaneous encroachment setting (denoted as superscript "ES") when the supplier engages in the marketplace as follows:

$$
\begin{aligned}
w^{\mathrm{ES}} & =-\frac{a\left[8+b\left(-8 \lambda+b\left(-4+b(1+\lambda)^{2}\right)\right)\right]}{2\left[-8+b^{2}(3+\lambda)\right]}, \\
q_{I}^{\mathrm{ES}} & =\frac{2 a(-1+b)}{-8+b^{2}(3+\lambda)}, \\
q_{S}^{\mathrm{ES}} & =-\frac{a[-8+b(2+b+b \lambda)]}{2\left[-8+b^{2}(3+\lambda)\right]}, \\
\prod_{I}^{\mathrm{ES}} & =\frac{a^{2}[16+64 c+b(-32+b(16-4 c(13+4 c)+b c(8+b(1+c)(5+c))))]}{4\left[-8+b^{2}(3+\lambda)\right]^{2}}-F, \\
\prod_{S}^{\mathrm{ES}} & =-\frac{a^{2}\left(12-8 c+b\left(-8+b(1+c)^{2}\right)\right)}{4\left[-8+b^{2}(3+\lambda)\right]^{2}}-F .
\end{aligned}
$$

According to the above equilibrium outcomes, the proof procedure is similar to Theorem 4.2. This concludes the proof.

Proof of Proposition 5.2. To begin with, we compare the values of $\lambda_{0}(b, F)$ and $\lambda_{2}(b, F)$, and $\lambda_{0}(b, F)>\lambda_{2}(b, F)$ always holds. Moreover, since we cannot characterize the relationships between $\lambda_{1}(b)$ and $\lambda_{3}(b)$, thereby we discuss it on two sides which have stated in the body of our paper. 
Proof of Theorem 5.3. We now present the equilibrium outcomes under price competition setting (denoted as superscript "EP") when the supplier engages in the marketplace as follows:

$$
\begin{aligned}
w^{\mathrm{EP}}= & -\frac{a(-1+\lambda)\left(8+b\left(-8 \lambda+b\left(-6+b+b^{2}+b \lambda(4+\lambda)\right)\right)\right.}{2\left(8+b^{4}-8 \lambda+b^{2}(-1+\lambda)(5+\lambda)\right)}, \\
p_{I}^{\mathrm{EP}}= & \frac{a\left(-12(-1+\lambda)+b\left(4(-1+\lambda)+b\left(-6+b+b^{2}-b \lambda(2+\lambda)+2 \lambda(3+\lambda)\right)\right)\right)}{2\left(8+b^{4}-8 \lambda+b^{2}(-1+\lambda)(5+\lambda)\right)}, \\
p_{S}^{\mathrm{EP}}= & -\frac{a\left(8-8 \lambda+b\left(2(1+\lambda)+b\left(-7-b(1+\lambda)+b^{2}(2+\lambda)+\lambda(2+\lambda)\right)\right)\right)}{2\left(8+b^{4}-8 \lambda+b^{2}(-1+\lambda)(5+\lambda)\right)}, \\
& a^{2}\left(8(-1+b)\left(-2+b^{2}\right)+(32+(-1+b) b(-96+b(-28+b(64+b(7+b(-18+b+\right. \\
\prod_{I}^{\mathrm{EP}}= & \frac{\left.\left.\left.\left.\left.2 b^{2}\right)\right)\right)\right)\right) \lambda+2\left(-2+b^{2}\right)\left(28+b(36+b(-18+b(-22+b(3+5 b)))) \lambda^{2}+(64+b(64+\right.}{4(1+b)\left(8+b^{4}-8 \lambda+b^{2}(-1+\lambda)(5+\lambda)\right)^{2}} \\
\prod_{S}^{\mathrm{EP}}= & -\frac{a^{2}(-1+\lambda)\left(12-8 \lambda+b\left(4-8 \lambda+b\left(-9+2 b^{2}+\lambda(4+\lambda)+b(-1+\lambda(4+\lambda))\right)\right)\right)}{4(1+b)\left(8+b^{4}-8 \lambda+b^{2}(-1+\lambda)(5+\lambda)\right)}-F .
\end{aligned}
$$

According to the above equilibrium outcomes, the proof procedure is similar to Theorem 4.2. This concludes the proof.

Acknowledgements. We thank the Editor(s) and the anonymous referees for their constructive and insightful comments, which help us significantly improve the quality of our paper. This paper was supported by the National Natural Science Foundation of China [Grant 72072041, 72001048, 71571052]; and the Chinese National Funding of Social Science (19BGL094).

\section{REFERENCES}

[1] V. Abhishek, K. Jerath and Z.J. Zhang, Agency selling or reselling? Channel structures in electronic retailing. Manage. Sci. 62 (2015) 2259-2280.

[2] A.M. Alaei, A. Taleizadeh and M. Rabbani, Marketplace, reseller, or web-store channel: the impact of return policy and cross-channel spillover from marketplace to web-store. J. Retail. Consum. Serv. (2020) 102271.

[3] A. Arya, B. Mittendorf and D. Sappington, The bright side of supplier encroachment. Market. Sci. 26 (2007) 651-659.

[4] N. Belhadj, D. Laussel and J. Resende, Marketplace or reselling? A signalling model. Inf. Econ. Policy 50 (2019) 100834.

[5] P. Chen, B. Li and H. Huang, Decision policies on players' different risk combination under supplier encroachment. RAIRO:OR 54 (2019) 1057-1075.

[6] M. Fleischmann, N. Agatz and J. Nunen, E-fulfillment and multi-channel distribution: a review. Eur. J. Oper. Res. 187 (2008) 339-356.

[7] H. Guan, H. Gurnani, X. Geng and Y. Luo, Strategic inventory and supplier encroachment. Manuf. Serv. Oper. Manage. 21 (2018) 536-555.

[8] A. Ha, X. Long and J. Nasiry, Quality in supply chain encroachment. Manuf. Serv. Oper. Manage. 18 (2016) $280-298$.

[9] A. Hagiu and J. Wright, Marketplace or reseller? Manage. Sci. 61 (2015) 184-203.

[10] S. Huang, X. Guan and Y.J. Chen, Retailer information sharing with supplier encroachment. Prod. Oper. Manage. 27 (2018) 1133-1147.

[11] S. Huang, S. Chen and X. Guan, Retailer information sharing under endogenous channel structure with investment spillovers. Comput. Ind. Eng. 142 (2020) 106346.

[12] D. Jia and S. Li, Optimal decisions and distribution channel choice of closed-loop supply chain when e-retailer offers online marketplace. J. Cleaner Prod. 265 (2020) 121767.

[13] Z. Li, S. Gilbert and G. Lai, Supplier encroachment under asymmetric information. Manage. Sci. 60 (2014) $449-462$.

[14] Z. Li, S. Gilbert and G. Lai, Supplier encroachment as an enhancement or a hindrance to nonlinear pricing. Prod. Oper. Manage. 24 (2015) 89-109.

[15] Y. Liu and Z. Zhang, Research notethe benefits of personalized pricing in a channel. Market. Sci. 25 (2006) $97-105$.

[16] J. Ma and Y. Hong, Research on manufacturer encroachment with advertising and design of incentive advertising: a gametheoretic approach. RAIRO:OR 55 (2021) S1261-S1286.

[17] B. Mantin, H. Krishnan and T. Dhar, The strategic role of third-party marketplaces in retailing. Prod. Oper. Manage. 23 (2014) 1937-1949. 
[18] B. Mittendorf, J. Shin and D.-H. Yoon, Manufacturer marketing initiatives and retailer information sharing. Quant. Market. Econ. 11 (2013) 263-287.

[19] J. Ryan, D. Sun and X. Zhao, Competition and coordination in online marketplaces. Prod. Oper. Manage. 21 (20126) $997-1014$.

[20] N. Singh and X. Vives, Price and quantity competition in a differentiated duopoly. RAND J. Econ. 15 (1984) 546-554.

[21] Y. Tan and J. Carrillo, Strategic analysis of the agency model for digital goods. Prod. Oper. Manage. 26 (2016) $724-741$.

$[22]$ L. Tian, A. Vakharia, Y. Tan and X. Yifan, Marketplace, reseller, or hybrid: strategic analysis of an emerging e-commerce model. Prod. Oper. Manage. 27 (2018) 1595-1610.

[23] M. Xue and J. Zhang, Supply chain encroachment with quality decision and different power structures. RAIRO:OR 54 (2020) 693-718.

[24] D.-H. Yoon, Supplier encroachment and investment spillovers. Prod. Oper. Manage. 25 (2016) $1839-1854$.

[25] J. Zhang, S. Li, S. Zhang and R. Dai, Manufacturer encroachment with quality decision under asymmetric demand information. Eur. J. Oper. Res. 273 (2019) 217-236.

\section{Subscribe to Open (S2O) A fair and sustainable open access model}

This journal is currently published in open access under a Subscribe-to-Open model (S2O). S2O is a transformative model that aims to move subscription journals to open access. Open access is the free, immediate, online availability of research articles combined with the rights to use these articles fully in the digital environment. We are thankful to our subscribers and sponsors for making it possible to publish this journal in open access, free of charge for authors.

\section{Please help to maintain this journal in open access!}

Check that your library subscribes to the journal, or make a personal donation to the $\mathrm{S} 2 \mathrm{O}$ programme, by contacting subscribers@edpsciences.org

More information, including a list of sponsors and a financial transparency report, available at: https://www. edpsciences.org/en/maths-s2o-programme 